\title{
A DIRECT EXTENSION OF MELLER'S CALCULUS
}

\author{
E.L. KOH \\ University of Petroleum and Minerals \\ Dhahran, Saudi Arabia \\ (Received June 26, 1980)
}

ABSTRACT. This paper extends the operational calculus of Meller for the operator $B_{\alpha}=t^{-\alpha} \frac{d}{d t} t^{\alpha+1} \frac{d}{d t}$ to the case where $\alpha \varepsilon(0, \infty)$. The development is à la Mikusinski calculus and uses Meller's convolution process with a fractional derivative operator.

KEY WORDS AND PHRASES. Operational Calculus, Mikusinski Calculus, Bessel Operator, Convolution Process, Fractional Derivative.

1980 MATHEMATICS SUBJECT CLASSIFICATION CODES. 44A40, 44A35, 33A40, 26A33, 93050.

\section{INTRODUCTION.}

Familiarity with integral transforms of distributions is assumed. This paper is accessible to readers familiar with references [1-3]. The generalist reader Interested in this area may start with these references.

Meller [4], [5] constructed an operational calculus for the operator $B_{\alpha}=t^{-\alpha} \frac{d}{d t} t^{\alpha+1} \frac{d}{d t}$ with $-1<\alpha<1$ by embedding it in a field of convolution quotients. The convolution process was given by the formula:

$$
\begin{aligned}
f(t) * g(t)=\frac{1}{\Gamma(1+\alpha) \Gamma(1-\alpha)} & \frac{d}{d t} \int_{0}^{t}(t-\xi)^{-\alpha} d \xi \frac{d}{d \xi} \xi \frac{d}{d \xi} \int_{0}^{\xi} d \eta x \\
\times & \int_{0}^{1} d x \eta^{\alpha}(1-x)^{\alpha} f(x n) g[(1-x)(\xi-n)] .
\end{aligned}
$$

This calculus reduces to Ditkin's calculus [6], [3] for $\frac{d}{d t} t \frac{d}{d t}$ when $\alpha=0$. Recently, Koh [7], [8] and Conlan [9] extended Meller's calculus to the case $\alpha \varepsilon(-1, \infty)$. A modified convolution process was used which yields results analogous to Meller's. In the present work, we give a direct extension of Meller's calculus by treating the operator $\frac{1}{\Gamma(1-\alpha)} \frac{d}{d t} \int_{0}^{1}(t-\xi)^{-\alpha} d \xi$ in $(1.1)$ as a fractional derivative. 
Specifically, we let $\mathrm{n}$ be the least integer greater than $\alpha \geq 0$. For any $\mathrm{n}-$ times differentiable function $f(t)$, the ath-order derivative of $f(t)$ is:

$$
D^{\alpha} f(t)=D^{n} I^{n-\alpha} f(t)
$$

where $D$ is $\frac{d}{d t}$ and $I^{\nu}$ is the Riemann-Liouville integral of order $\nu>0$ given in Ross [10] by

$$
I^{\nu} f(t)=\frac{1}{\Gamma(\nu)} \int_{0}^{t}(t-\xi)^{\nu-1} f(\xi) d \xi
$$

It is easy to see that $I^{\nu}$ satisfies the semigroup property

$$
I^{\alpha} I^{\beta}=I^{\alpha+\beta}
$$

but $D^{\alpha}$ does not. Thus $D^{\alpha} D$ in (2.1) below cannot be written $D^{\alpha+1}$.

\section{THE CONVOLUTION QUOTIENTS.}

Let $\alpha \geq 0$ be a fixed real number. Let $C^{\infty}$ denote the linear space of infinite1y differentiable functions on $[0, \infty)$. For every pair of functions $\phi(t)$ and $\psi(t)$ in $\mathrm{C}^{\infty}$, define their convolution by

$$
\phi(t) * \psi(t)=\frac{1}{\Gamma(\alpha+1)} D^{\alpha} D t D \int_{0}^{t} \int_{0}^{1} n^{\alpha}(1-x)^{\alpha} \phi(x n) \psi[(1-x)(t-n)] d x d n .
$$

From this definition, the following properties are clear: (i) $C^{\infty}$ is closed under convolution, (ii) convolution is bilinear on $C^{\infty} \times C^{\infty}$, (iii) convolution is distributive with respect to the usual addition of functions. It also follows immediately that equation (2.1) specilizes to Meller's convolution for $\alpha<1$ and to Ditkin's for $\alpha=0$. Not so immediate are the following properties.

PROPOSITION 1. Convolution is commutative.

PROOF. Let $x=1-\frac{v}{t}$ and $\eta=t-t \xi$ in (2.1) and noting that the Jacobian $\frac{\partial(x, \eta)}{\partial(v, \xi)}=1$ for all $t \varepsilon(0, \infty)$, we have

$$
\begin{aligned}
\phi(t) * \psi(t) & =\frac{1}{\Gamma(\alpha+1)} D^{\alpha} D t D \int_{0}^{1} \int_{0}^{t}(t-t \xi)^{\alpha}\left(\frac{v}{t}\right)^{\alpha} \phi\left[\left(1-\frac{v}{t}\right)(t-t \xi)\right] \psi\left[\frac{v}{t}(t \xi)\right] d v d \xi \\
& =\frac{1}{\Gamma(\alpha+1)} D^{\alpha} D t D \int_{0}^{t} \int_{0}^{1} v^{\alpha}(1-\xi)^{\alpha} \psi(\xi v) \phi[(1-\xi)(t-v)] d \xi d v \\
& =\psi(t) * \phi(t) .
\end{aligned}
$$

PROPOSITION 2. For every complex number $\lambda$, and any $\phi(t) \varepsilon C^{\infty}, \lambda \star \phi(t)=\lambda \phi(t)$. PROOF. $\quad \lambda * \phi(t)=\frac{1}{\Gamma(\alpha+1)} D^{\alpha} D t D \int_{0}^{t} \eta^{\alpha} \int_{0}^{1}(1-x)^{\alpha} \lambda \phi(x n) d x d \eta$

$$
=\frac{\lambda}{\Gamma(\alpha+1)} D^{\alpha} D^{\alpha+1} \int_{0}^{1}(1-x)^{\alpha} \phi(x t) d x
$$




$$
\begin{aligned}
& =\frac{\lambda}{\Gamma(\alpha+1)} D^{\alpha} D \int_{0}^{t}(t-u)^{\alpha} \phi(u) d u \\
& =\frac{\lambda}{\Gamma(\alpha+1)} D^{n} I^{n-\alpha} \int_{0}^{t} \alpha(t-u)^{\alpha-1} \phi(u) d u \\
& =\lambda D^{n} I^{n-\alpha} I^{\alpha} \phi(t)=\lambda \phi(t) .
\end{aligned}
$$

The last step follows from (1.4) and (1.2). q.e.d. In view of Proposition 2, there is no distinction between constants and constant functions in our calculus.

PROPOSITION 3. Convolution is associative.

PROOF. A direct calculation shows that, for nonnegative integers $q$ and $r$,

$$
t^{q} t^{r}=\frac{q ! r ! \Gamma(q+\alpha+1) \Gamma(r+\alpha+1)}{(q+r) ! \Gamma(\alpha+1) \Gamma(q+r+\alpha+1)} t^{q+r} .
$$

Hence on using (2.2) again,

$$
\begin{aligned}
t^{p} *\left(t^{q} t_{t}\right) & =\frac{p ! q ! r ! \Gamma(p+\alpha+1) ! \Gamma(q+\alpha+1) \Gamma(r+\alpha+1)}{(p+q+r) ! \Gamma(\alpha+1) \Gamma(p+q+r+\alpha+1) \Gamma(\alpha+1)} t^{p+q+r} \\
& =\left(t^{p} * t^{q}\right) * t^{r} .
\end{aligned}
$$

Due to the bilinearity of our convolution, equation (2.3) still holds for polynom1als. Our proposition follows from Weierstrass's Approximation Theorem and the fact [9] that the space of $C^{\infty}$ functions with compact support is dense in $C^{\infty} \cdot q \cdot e \cdot d$.

PROPOSITION 4. $\mathrm{C}^{\infty}$ has no zero divisors, i.e. if $\phi(t)$ and $\psi(t)$ belong to $C^{\infty}$ and $\phi(t) * \psi(t)=0$, then either $\phi(t)=0$ or $\psi(t)=0$.

PROOF. $\phi(t) * \psi(t)=0$ implies that

$$
\begin{aligned}
\int_{0}^{t}(t-\xi)^{n-\alpha-1} \frac{d}{d \xi} \xi & \frac{d}{d} \int_{0}^{\xi} \int_{0}^{1} n^{\alpha}(1-x)^{\alpha} \phi(x n) \psi[(1-x)(\xi-n)] d x d n \\
& =c_{1} \frac{t^{n-1}}{(n-1) !}+c_{2} \frac{t^{n-2}}{(n-2) !}+\cdots \cdot+c_{n} .
\end{aligned}
$$

As $t \rightarrow 0, c_{n}=0$. Now, by an argument leading to (2.3), we see that, if $c_{1} \neq 0$ for some 1 , then $\phi(t)$ and $\psi(t)$ have to be polynomials. But if they are polynomials, the left side of (2.4) will be of degree at least $n$. Hence, the right side of (2.4) has to be zero. A similar argument, together with Titchmarsh's Theorem [2], yields

$$
\int_{0}^{t} \int_{0}^{1} \eta^{\alpha}(1-x)^{\alpha} \phi(x \eta) \psi[(1-x)(t-\eta)] d x d \eta=0 .
$$


To complete the proof, let $x=\frac{y}{\tau}, \eta=z \tau$ and $t=v \tau$ in $(2.5)$. We then have

$$
\int_{0}^{v} \int_{0}^{1} z^{\alpha}(\tau-y)^{\alpha} \phi(y z) \psi[(\tau-y)(v-z)] d y d z=0 .
$$

By a theorem of Mikusinski and Ryll-Nardzewski [11], it follows that $z^{\alpha} \phi(y z)=0$ or $y^{\alpha} \psi(y z)=0$. Thus, $\phi(t)=0$ or $\psi(t)=0$.

q.e.d.

The above properties establish $\mathrm{C}^{\infty}$ as an integral domain under the operations of addition and convolution as multiplication. By virtue of Proposition 2, the multiplicative identity for $C^{\infty}$ is the number 1 . We may now extend $c^{\infty}$ into the field $F$ of convolution quotients consisting of equivalence classes of ordered pairs $(\phi, \psi)$ of elements in $c^{\infty}$ with $\psi \neq 0$. The equivalence relation is given by

$$
\left(\phi_{1}, \psi_{1}\right) \sim\left(\phi_{2}, \psi_{2}\right) \text { if } \phi_{1} * \psi_{2}=\phi_{2} * \psi_{1}
$$

As usual, convolution quotients are called operators [2] and are denoted by $\frac{\phi}{\psi}$. Operators of the form $\frac{\phi(t)}{1}$ constitute a subring of $F$ isomorphic to $c^{\infty}$ through the canonical maps $\frac{\phi(t)}{1} \leftrightarrow \phi(t)$.

3. AN OPERATIONAL CALCULUS.

We now show that the operator $B_{\alpha}$ belongs to $F$. First, note that a right inverse to $B_{\alpha}$ is given by

$$
\Lambda \phi=\int_{0}^{t} \xi^{-\alpha-1} \int_{0}^{\xi} \eta^{\alpha} \phi(\eta) d n d \xi
$$

1.e., $B_{\alpha} \Lambda \phi=\phi$, for $\phi \varepsilon C^{\infty}$. If we restrict the domain of $B_{\alpha}$ to $\left\{\phi \varepsilon C^{\infty} \mid \phi(0)=0\right\}$, then $\Lambda$ is also a left inverse; 1.e., $\Lambda B_{\alpha} \phi=\phi$.

PROPOSITION 5. For any $\phi(t) \varepsilon C^{\infty}, \frac{t}{\alpha+1} * \phi(t)=\Lambda \phi(t)$.

PROOF. We shall assume that $\alpha$ is not an integer. Otherwise, the proof is more straightforward, obviating the use of fractional integrals.

$$
\begin{aligned}
& \frac{t}{\alpha+1} * \phi(t)=\frac{1}{\Gamma(\alpha+1)} D^{\alpha} D t D \int_{0}^{t} \int_{0}^{1} n^{\alpha}(1-x)^{\alpha} \phi(x) \frac{(1-x)(t-\eta)}{\alpha+1} d x d \eta \\
& =\frac{1}{\Gamma(\alpha+2)} D^{\alpha} D t D \int_{0}^{t} \frac{(t-\eta)}{\eta^{2}} \int_{0}^{n}(\eta-\xi)^{\alpha+1} \phi(\xi) d \xi d \eta \\
& =\frac{1}{\Gamma(\alpha+2)} D^{\alpha}\left\{\int_{0}^{t} \frac{1}{\eta^{2}} \int_{0}^{\eta}(\eta-\xi)^{\alpha+1} \phi(\xi) d \xi d \eta+\frac{1}{t} \int_{0}^{t}(t-\xi)^{\alpha+1} \phi(\xi) d \xi\right\} \\
& =\frac{1}{\Gamma(\alpha+1)} D^{\alpha} \int_{0}^{t} \frac{1}{n} \int_{0}^{n}(n-\xi)^{\alpha} \phi(\xi) \mathrm{d} \xi \mathrm{d} n \text {. }
\end{aligned}
$$


Let $\psi(\xi)=I^{n} \phi(\xi)$ where $\mathrm{n}=$ least integer greater than $\alpha$. Then (3.1) becomes

$$
\begin{aligned}
\frac{t}{\alpha+1} * \phi(t) & =D^{\alpha} \int_{0}^{t} \frac{1}{n} \int_{0}^{n} \frac{(n-\xi)^{\alpha-n}}{\Gamma(\alpha-n+1)} \psi(\xi) d \xi d n \\
& =D^{n} \int_{0}^{t} \frac{(t-u)^{n-\alpha-1}}{(n-\alpha)} \int_{0}^{u} \frac{1}{n} \int_{0}^{n} \frac{(n-\xi)^{\alpha-n}}{\Gamma(\alpha-n+1)} \psi(\xi) d \xi d n d u \\
& =\frac{D^{n}}{\Gamma(n-\alpha+1) \Gamma(\alpha-n+1)} \int_{0}^{t} \psi(\xi) \int_{\xi}^{t} n^{-1}(n-\xi)^{\alpha-n}(t-n)^{n-\alpha} d n d \xi .
\end{aligned}
$$

The inner integral reduces, via the Beta function, to $\left\{\left(\frac{\xi}{t}\right)^{\alpha-n}-1\right\} \Gamma(\alpha-n+1) \Gamma(n-\alpha)$. Thus,

$$
\begin{aligned}
\frac{t}{\alpha+1} * \phi(t) & =\frac{D^{n}}{n-\alpha} \int_{0}^{t} \psi(\xi)\left\{\left(\frac{\xi}{t}\right)^{\alpha-n}-1\right\} d \xi \\
& =\frac{D^{n}}{n-\alpha} \int_{0}^{1} t \psi(w t)\left(w^{\alpha-n}-1\right) d w \\
& =\int_{0}^{1}\left(\frac{w^{\alpha-n}-1}{n-\alpha}\right)\left(w^{n} t \phi(w t)+n w^{n-1} I \phi(w t)\right) d w \\
& =\int_{0}^{t} \frac{1}{n-\alpha}\left[\left(\frac{\xi}{t}\right)^{\alpha}-\left(\frac{\xi}{t}\right)^{n}\right] \phi(\xi) d \xi \\
& =\int_{0}^{t} \frac{\phi(\xi)}{-\alpha}\left[\left(\frac{\xi}{t}\right)^{\alpha}-1\right] d \xi \\
& =\int_{0}^{t} \xi^{\alpha} \phi(\xi) \int_{0}^{t} n^{-\alpha-1} d n d \xi \\
& =\int_{0}^{t} n^{-\alpha-1} \int_{0}^{n} \xi^{\alpha} \phi(\xi) d \xi d n=\Lambda \phi(t) .
\end{aligned}
$$

This result implies that operators of the form $\frac{\phi(t)}{t}$ with $\phi(0)=0$ may be identified with locally integrable functions $f(t)$ such that $\Lambda f(t)<\infty$ for every $t>0$. Indeed,

$$
\frac{\phi(t)}{t}=f(t) \varepsilon L_{l o c}[0, \infty) \text { iff } \phi(t)=t * f(t)=(\alpha+1) \Lambda f<\infty, \quad v t>0 .
$$

The next result follows from Proposition 5 and Equation (2.2) by induction.

PROPOSITION 6. Let $k$ be a positive integer. Then, for any $\phi(t) \varepsilon c^{\infty}$,

$$
\left.\frac{n ! t^{k}}{(k+n) ! k !} * \phi(t)=\Lambda^{k} \phi(t) \text { where } \Lambda^{k} \phi(t)=\underset{k-t i m e s}{\Lambda(\Lambda(\cdots)}\right) .
$$

Let $\mathrm{v}$ be the operator $\frac{\alpha+1}{t}$ and $v^{k}$ the $k$-times application of $v$. 
PROPOSITION 7. For any $2 k$ times differentiable function $\phi(t)$,

$$
v^{k} \phi(t)=B_{\alpha}^{k} \phi(t)+\left.\sum_{j=1}^{k} B_{\alpha}^{k-j} \phi(t)\right|_{t \rightarrow 0^{+}} v^{j}
$$

PROOF. $\phi(t)=\Lambda_{\alpha} \phi(t)+\phi(0)=\frac{t}{\alpha+1} * B_{\alpha} \phi(t)+\phi(0)$. Thus, $\mathrm{V} \phi(\mathrm{t})=\mathrm{B}_{\alpha} \phi(\mathrm{t})+\phi(0) \mathrm{V}$ and (3.2) is proved for $\mathrm{k}=1$. Suppose now that (3.2) is true for $k=m-1$. Then for any $2 m$ times differentiable function $\phi(t)$,

$$
\begin{aligned}
v^{m} \phi(t) & =v\left(B_{\alpha}^{m-1} \phi(t)+\left.\sum_{j=1}^{m-1} B_{\alpha}^{m-1-j} \phi(t)\right|_{t \rightarrow 0^{+}} v^{j}\right) \\
& =B_{\alpha} B_{\alpha}^{m-1} \phi(t)+\left.B_{\alpha}^{m-1} \phi(t)\right|_{t \rightarrow 0^{+}} v+\left.\sum_{j=1}^{m-1} B_{\alpha}^{m-1-j} \phi(t)\right|_{t \rightarrow 0^{+}} v^{j+1} \\
& =B_{\alpha}^{m} \phi(t)+\left.\sum_{j=1}^{m} B_{\alpha}^{m-j} \phi(t)\right|_{t \rightarrow 0^{+}} v^{j} .
\end{aligned}
$$

The proposition follows by induction.

A number of operational formulas such as those in Theorems 5 and 6 of [7] may be generated by using (3.2). The proofs are similar, mutatis mutandis. A generalization of Theorem 5 of [7] is obtained by parametric differentiation.

$$
\text { PROPOS ITION 8. } \begin{aligned}
\frac{V}{(V-a)^{m+1}} & =\frac{\Gamma(\alpha+1)}{m !} t^{m}(a t)^{-\frac{\alpha+m}{2}} I_{\alpha+m}(2 \sqrt{a t)} \\
\frac{V}{(V+a)^{m+1}} & =\frac{\Gamma(\alpha+1)}{m !} t^{m}(a t)^{-\frac{\alpha+m}{2}} J_{\alpha+m}(2 \sqrt{a t)}
\end{aligned}
$$

where $I_{V}(x)$ and $J_{V}(x)$ are Bessel functions of order $v$.

REMARKS. 1. All the results of Meller are extendible to the case $\alpha \varepsilon(0, \infty)$ via the methoo given in this paper.

2. The operational calculus may be applied to certain time-varying systems and to Kratzel's problem as done in [8].

3. In [12], a convolution for the operator $\Delta=t^{-n-1} \frac{d}{d t} t^{n+1} \frac{d}{d t}$, where $n$ is a natural number, is given which is associative, commutative, and distributive with respect to addition. However, the rirg under convolution as multiplication contains zero divisors.

ACKNOWLEDGEMENT. The author is on leave of absence from the University of Regina, Regina, Canada. The research is partly supported by the National Research Council of Canada under a grant numbered A-7184. 


\section{REFERENCES}

1. ZEMANIAN, A.H. Distribution Theory and Transform Analysis, McGraw-Hi11, New York, 1965.

2. MIKUSINSKI, J. Operational Calculus, Pergamon Press, Oxford, 1959.

3. DITKIN, V.A. and PRUDNIKOV, A.P. Integral Transforms and Operational Calculus, Pergamon Press, New York, 1965.

4. MELLER, N.A. On an operational calculus for the operator $B_{\alpha}=t^{-\alpha \frac{d}{d t}} t^{\alpha+1} \frac{d}{d t}$, Vichishitelnaya Matematika $\underline{6}$ (1960), 161-168.

5. MELLER, N.A. Some applications of operational calculus to problems in analysis, Zh. Vichisl. Mat. 1. Mat. Fiz. 3(1) (1963), 71-89.

6. DITKIN, V.A. Operational calculus theory, Dok1. Akad. Nauk. SSSR. 116 (1957), 15-17.

7. KOH, E.L. A Mikusinski calculus for the Bessel operator $B_{\mu}$, Proc. Diff. Eq., Springer-Verlag Lect. Notes \#564 (1976), 291-300.

8. $\mathrm{KOH}$, E.L. Application of an operational calculus to certain time-varying systems, Int. J. Systems Sc. 9 (1978), 595-601.

9. CONLAN, J. and $\mathrm{KOH}$, E.L. On the Meijer transformation, Int. J. Math. and Math. Sc. 1 (1978), 145-159.

10. ROSS, B. (ed.) Fractional Calculus and its Applications, Springer-Verlag, Berlin, 1975.

11. MIKUSINSKI, J. and RYLL-NARDZEWSKI, C. Un theorèmé sur le product de composition des fonctions de plusieurs variables, Studia Math. 13(1) (1953), 62-68.

12. CHOLEWINSKI, F.M. A Hankel convolution complex inversion theory, A.M.S. Memoirs $\$ 58$, Providence, 1965. 


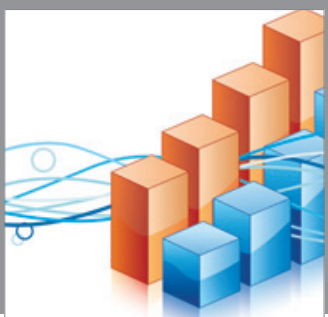

Advances in

Operations Research

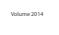

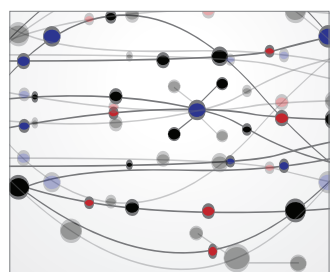

\section{The Scientific} World Journal
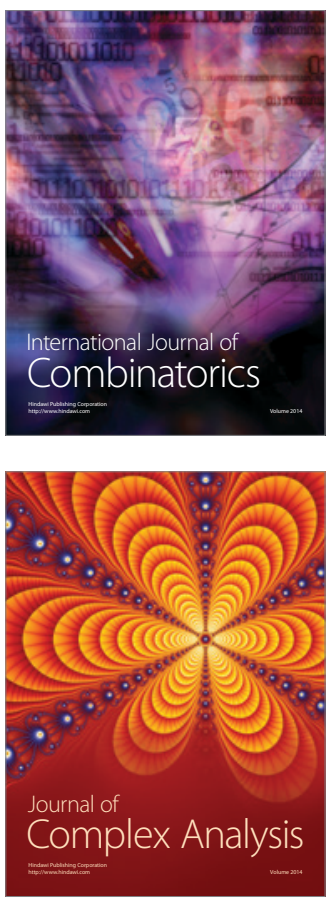

International Journal of

Mathematics and

Mathematical

Sciences
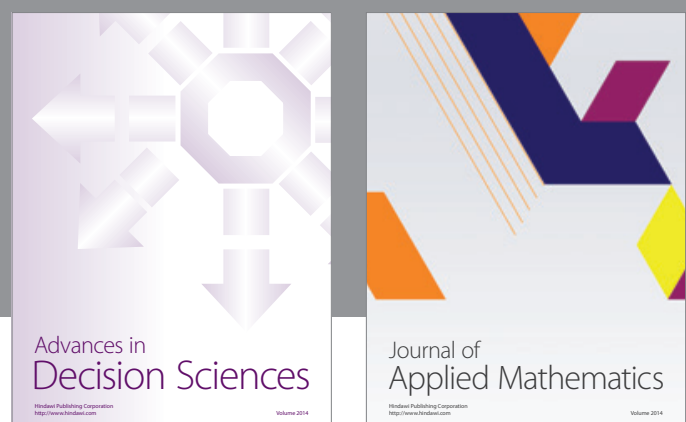

Journal of

Applied Mathematics
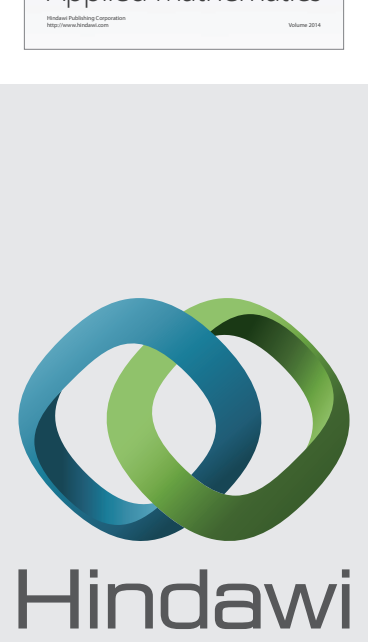

Submit your manuscripts at http://www.hindawi.com
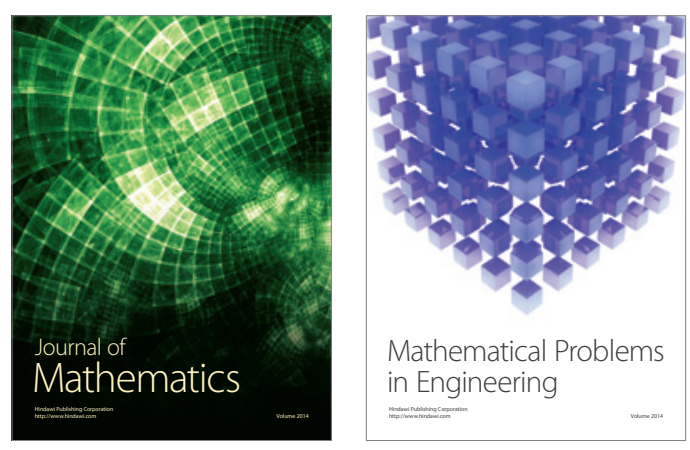

Mathematical Problems in Engineering
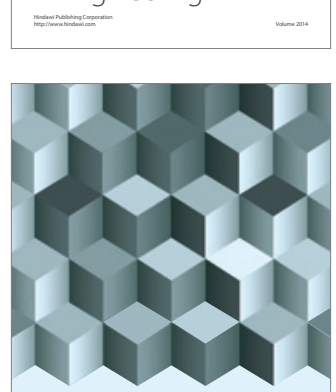

Journal of

Function Spaces
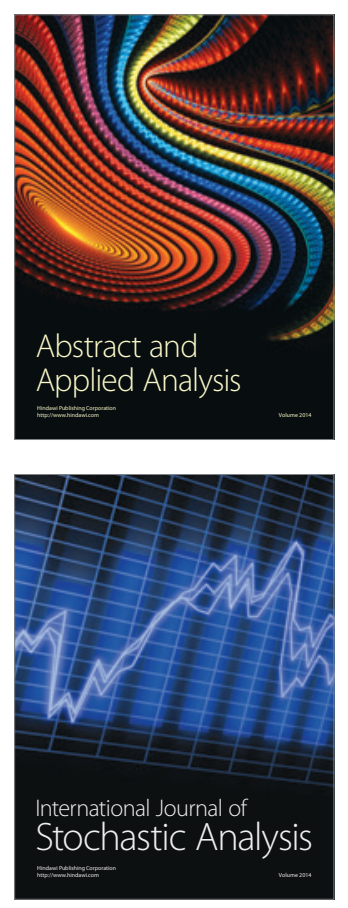

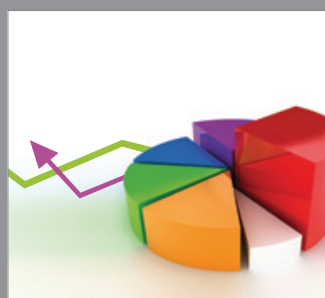

ournal of

Probability and Statistics

Promensencen
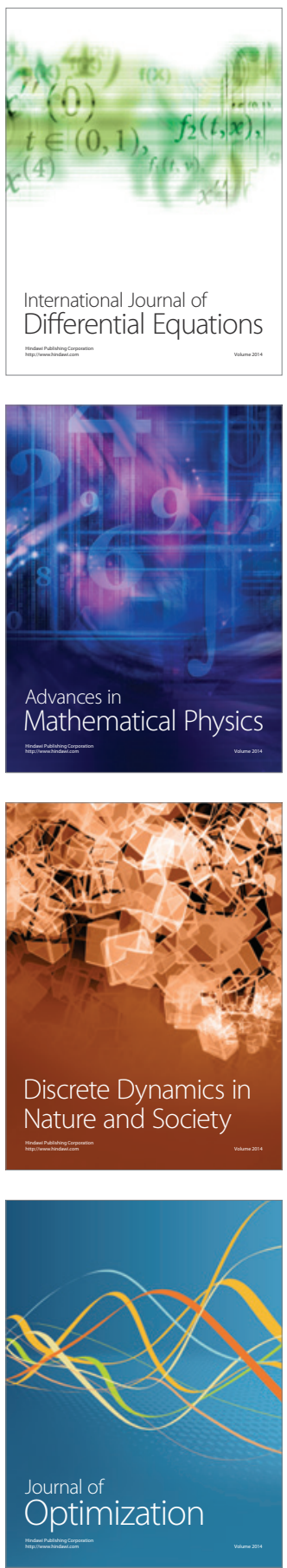